\title{
ANÁLISIS DE LA EXTINCIÓN DE LA POLÍTICA PÚBLICA DE DESTINOS INDUCTORES DEL DESARROLLO DEL TURISMO REGIONAL EN BRASIL
}

\author{
Magnus Luiz Emmendoerfer \\ Universidad Federal de Viçosa, Brasil \\ magnus@ufv.br \\ Fábia Tretin \\ Universidad Federal Fluminense, Brasil \\ Thiago Duarte Pimentel \\ Universidad Federal de Juiz de Fora, Brasil \\ María Belén Zambrano-Pontón \\ Universidad Federal de Viçosa, Brasil
}

\section{Resumen}

Este artículo analizó el término del proyecto gubernamental - Destinos Inductores del Desarrollo del Turismo Regional - DIDTR en Brasil, principal política pública nacional del turismo (2008-2015). El marco teórico se basó en las Políticas Públicas de Turismo y en la fase de Extinción del Ciclo Político. Para este estudio, se utilizó un abordaje empírico cualitativo. Las unidades de análisis fueron los destinos turísticos de Minas Gerais. Así, el método utilizado fue el estudio de caso (Proyecto DIDTR), cuyos datos fueron colectados por medio de entrevistas con stakeholders del sector turístico. Los principales resultados mostraron que una política pública puede terminar por motivos hasta hoy no tratados por la comunidad científica. Para este caso específico, fueron mostrados dos momentos, el primero para fines internos, en 2011, que tuvo como principal causa, los cortes presupuestarios, como resultado de una investigación de corrupción, específicamente, desvío de dinero proveniente de enmiendas parlamentarias, que involucraron al Ministerio del Turismo (MTur). El segundo momento para fines externos, en 2015, con el final de la acción más emblemática, la emisión de los informes de los índices de competitividad. Finalmente, ninguno de los momentos de finalización del proyecto fue debidamente esclarecido y comunicado por el MTur a los destinos involucrados.

Palabras Claves: Extinción de Políticas Públicas, Géstion Pública, Destinos Inductores de Turismo, Brasil.

Cita Recomendada: Emmendoerfer, M.; Tretin, F.; Pimentel, T. D. \& Zambrano-Pontón , M. (2019). Análisis de la extinción de la política pública de Destinos Inductores del Desarrollo del Turismo Regional en Brasil. Revista Gestión Turística, (32), 08-28. ISSN 0717-1811. 
PUBLIC POLICY TERMINATION ANALYSIS OF INDUCTIVE DESTINATIONS OF THE DEVELOPMENT TOURISM REGIONAL IN BRAZIL

Magnus Luiz Emmendoerfer

Universidad Federal de Viçosa, Brasil magnus@ufv.br

Fábia Tretin

Universidad Federal Fluminense, Brasil

Thiago Duarte Pimentel

Universidad Federal de Juiz de Fora, Brasil

María Belén Zambrano-Pontón

Universidad Federal de Viçosa, Brasil

\begin{abstract}
This paper researched the ending of governmental Project called Inductive Destinations of the Development Tourism Regional (DIDTR) in Brazil, the main national public policy on tourism (2008-2015). The theoretical framework was based on the Tourism Public Policies and the phase of ending of the Political Cycle. For this study, it was used a qualitative empirical approach, the tourist destinations of Minas Gerais. Additionality, the case study method was applied (DIDTR Project) in this study using interviews with the principal stakeholders of the tourism sector. The main results of the study showed that a policy public can finalize with reasons until now no studied by the Scientific Community. For this specific case, it was shown two moments, the first for internal purposes in 2011, that had as the main cause, the budgetary cuts as a result of the corruption investigations, specifically the misappropriation of money from parliamentary amendments which involved to Ministry of Tourism (MTur). The second moment for external purposes in 2015, with the final of the most emblematic action, the issuance of the competitiveness indices reports. Finally, none of the moments of the Project termination was officially communicated by the MTur to the touristic destinations involved.
\end{abstract}

Keywords: Ending of Public Policies, Public Management, Tourism-Inductive Destinations, Brazil.

Recommended Cite:-Emmendoerfer, M.; Tretin, F.; Pimentel, T. D. \& Zambrano-Pontón, M. (2019). Análisis de la extinción de la política pública de Destinos Inductores del Desarrollo del Turismo Regional en Brasil. Revista Gestión Turística, (32), 08-28. ISSN 0717-1811. 


\section{Introducción}

Mejorar la calidad de vida de la población constituye una de las premisas que persiguen, o deberían perseguir, los gobiernos en todo el mundo y es así, que los Estados pueden intervenir, para alcanzar ese bienestar a través de las políticas públicas, las cuales pueden ser observadas y analizadas utilizando el modelo del ciclo político. El ciclo o proceso político es uno de los modelos más utilizados para el análisis de las políticas públicas, inicialmente propuesto por Lasswell (1956), el autor presenta el modelo en siete pasos: inteligencia, promoción, prescripción, innovación, aplicación, conclusión y examen (evaluación).

A partir de entonces, varios autores proponen tipologías con diferentes números de fases de la propuesta inicial. Para Secchi (2013), por ejemplo, el ciclo presenta siete etapas: 1. Identificación del Problema, 2. Formación de la Agenda, 3. Formulación de las alternativas, 4. Toma de Decisión, 5. Implementación, 6. Evaluación y 7. Extinción, cerrando el ciclo con esta etapa. Subirats (1989) resalta la importancia de que se analicen los programas realizados por la administración pública, analizando así, los efectos que el programa dejó, para el autor una intervención gubernamental no puede acabarse con la evaluación de sus resultados, es preciso cerrar el ciclo.

En Brasil, Secchi (2013) otorga especial atención a la etapa final del ciclo político $\mathrm{y}$, en este artículo resaltamos que para el sector del turismo no hay evidencias producidas por la comunidad científica y técnica sobre los motivos de la extinción de una política pública, siendo muy importante analizar y comprender las razones que llevan a un gobierno a decidir el final de las políticas públicas, específicamente políticas relacionadas al turismo. De acuerdo con Souza y Secchi (2015) entre los principales motivos que pueden influenciar en la decisión de los gobiernos para llegar a extinguir una política pública están razones referentes al problema, a la solución de la política y al ambiente político.

En ese sentido, la pregunta que guió esta investigación fue: ¿Cuáles son las razones para la extinción de políticas públicas, programas o proyectos de desarrollo turístico regional en municipios considerados inductores de turismo?

En ese sentido, los países desarrollan políticas y estrategias propias o adaptadas a su realidad, con la intención de promover e incentivar el turismo en las 
perspectivas económicas, sociales y hasta políticas y de ahí la importancia de la intervención del Estado, a través de políticas públicas que ayuden a coordinar y dirigir la actividad turística. La intervención del Estado en el turismo, de acuerdo con Castilho y Selva (2012), busca atender varios objetivos como el desarrollo socioeconómico, prosperidad local, generación de trabajo y renta, seguridad pública, sustentabilidad, bienestar comunitario, competitividad, hospitalidad y ordenamiento territorial.

Así, el interés por el estudio de las políticas públicas vinculadas a los destinos turísticos ha ido en aumento, eso puede asociarse al posible desarrollo socioeconómico que puede acarrear la actividad turística en Brasil y el mundo entero (Emmendoerfer \& Soares, 2014). En Brasil, dentro de la Política Nacional propuesta por el Gobierno Federal en materia de turismo, está el objeto de estudio de este trabajo, el proyecto "Destinos Inductores del Desarrollo del Turismo Regional" - DIDTR, elaborado por el Ministerio del Turismo - MTur, en 2008, y que aparentemente, para la sociedad y los destinos, terminó en 2015. Para fines de este artículo, se entiende el proyecto como un conjunto de acciones; programa como un conjunto de proyectos; $\mathrm{y}$, políticas como un conjunto de programas, ejecutados de modo Estado-céntrico o Socio-céntrico a

Siendo así, este trabajo analizó los motivos que llevaron al gobierno federal brasileño a decidir extinguir la principal política pública nacional de turismo conocida como Destinos Inductores del Desarrollo del Turismo Regional - DIDTR en el periodo de 2008-2015.

\section{Marco teórico}

La literatura científica especializada sobre políticas públicas y turismo es muy reciente. Las bases teóricas son principalmente de la ciencia política y de la administración pública. Esta breve revisión focalizó el tema extinción de política pública que aún más rara su discusión en la comunidad científica, especialmente, en los estudios del turismo y sobre cuestiones de la América Latina.

De acuerdo con Lynn y Gould (1980), las políticas públicas son todas las acciones específicas del gobierno que buscan que los efectos producidos sean también específicos. Para Araújo y Rodríguez (2017) las políticas públicas constituyen procesos complejos que se desarrollan en diversos niveles de actuación y de- 
cisión, pudiendo ser: local, regional, nacional, o transnacional; involucrando a varios actores: gobernantes, legisladores, electores, administración pública, grupos de interés y organismos internacionales, que actúan en contextos específicos, buscando solucionar problemas públicos.

Así, toda política pública puede ser analizada a través del modelo del ciclo político, dicho modelo bajo la perspectiva de Frey (2009, p. 226), supone que "al subdividir el accionar público en fases parciales del proceso político-administrativo de resolución de problemas, el policy cycle se revela como un modelo heurístico muy interesante para el análisis de la vida de una política pública”.

La extinción constituye la etapa final de las políticas públicas, aunque no todas lleguen a concluir el policy cycle (Secchi, 2013). La fase de Extinción que para fines de este artículo constituye la etapa de término de una o más acciones que componen la política pública, siendo varias las causas estudiadas hasta hoy, de acuerdo con Giuliani (2005) los motivos más comunes para que exista la extinción de una política pública, son: 1. Que se perciba que el problema público, para el que fue creada la política pública sea percibido como resuelto; 2. Que sean percibidos como ineficaces los programas y acciones de la formulación de alternativas; 3. En casos en que, aunque el problema no haya sido resuelto, perdió importancia para continuar bajo la protección del Estado.

El estudio de las políticas públicas enfocadas en el ciclo político y, particularmente, la fase de extinción es un tema que ha sido analizado desde la década de 1970, principalmente, en los trabajos realizados por la academia internacional. En los estudios realizados en Brasil se evidencia de alguna manera un "olvido" de la fase de extinción, siendo que ella podría constituir una etapa esencial para la toma de futuras decisiones y, considerando que, de la misma forma como ocurre con cualquier tipo de organización, las políticas públicas también pueden llegar a su fin, siendo necesario comprender los motivos que llevan a ese cierre (Souza \& Secchi, 2015).

De ahí la importancia de conocer las razones de la extinción de las políticas públicas, siendo los motivos muy diversos. En la visión de DeLeon (1977), son dos posibilidades para esa extinción: 1. cuando el problema público, por el cual fue creada la política fue percibido como resuelto; 2 . cuando el problema creció, haciendo que la continuidad de la política no sea posible más. Existen, también, 
políticas públicas que fueron creadas con una fecha de validad determinada, siendo la extinción planeada con anticipación.

De acuerdo como presentado por Souza y Secchi (2015), entre las principales razones que pueden ejercer influencia en la decisión de los gobiernos para llegar a la extinción de una política pública están las relativas al problema, a la solución de la política y al ambiente político. (Ver Tabla 1.)

Tabla 1. Razones y percepciones para la extinción de una política pública

\begin{tabular}{|c|c|}
\hline $\begin{array}{c}\text { Razones para la ex- } \\
\text { tinción }\end{array}$ & Percepciones \\
\hline \multirow{4}{*}{$\begin{array}{c}\text { Razones relativas al } \\
\text { problema }\end{array}$} & Problema resuelto \\
\hline & Problema agravado \\
\hline & Atención al problema disminuido \\
\hline & Cambio en la atención de la naturaleza del problema \\
\hline \multirow{5}{*}{$\begin{array}{l}\text { Razones relativas a } \\
\text { la solución }\end{array}$} & Política Pública Ineficaz \\
\hline & Política Pública Eficaz \\
\hline & Política Pública con plazo expirado \\
\hline & Política Pública sustituida \\
\hline & Política pública incorporada \\
\hline \multirow{5}{*}{$\begin{array}{l}\text { Razones relativas al } \\
\text { ambiente político }\end{array}$} & Presión de los medios y de la opinión pública \\
\hline & Cambio en la administración y en el Gobierno \\
\hline & Ideología Política \\
\hline & Cuestiones financieras \\
\hline & Eficiencia organizacional \\
\hline
\end{tabular}

Fuente: Adaptado de Souza y Secchi (2015, p.89). Traducción nuestra.

No obstante, extinguir políticas públicas no es un trabajo fácil, ya que existen obstáculos que pueden llegar hasta a prohibir la extinción de las mismas (Souza \& Secchi, 2015). Obstáculos de diversa naturaleza como legal, administrativo, cultural y hasta moral. 
Análisis de la extinción de la política pública de destinos inductores del desarrollo del turismo regional en Brasil. Gestión Turística Nº 32 julio-diciembre 2019, pp 08-28.
Magnus Emmendoerfer Fábia Tretin Thiago D. Pimentel Ma. Belén Zambrano-Pontón

\section{Procedimientos metodológicos}

Esta investigación tuvo un enfoque cualitativo de tipo descriptivo. La estrategia de investigación fue el estudio de caso del proyecto DIDTR, cuyas unidades de análisis contemplaron los cuatro municipios, considerados destinos inductores nacionales del estado de Minas Gerais en Brasil: Belo Horizonte, Ouro Preto, Diamantina y Tiradentes. El proyecto DIDTR contemplaba 65 ciudades designadas como destinos inductores nacionales de turismo, como se muestra en la Figura 1. Según el Ministério do Turismo (2009), para ser escogidos como destinos inductores en Brasil, fueron considerados los municipios que por lo menos tenían una infraestructura turística básica, sus atractivos calificados y capacidad para recibir y generar turistas para otros destinos.

Figura 1. Mapa de los 65 Destinos Inductores de Desarrollo Turístico Regional

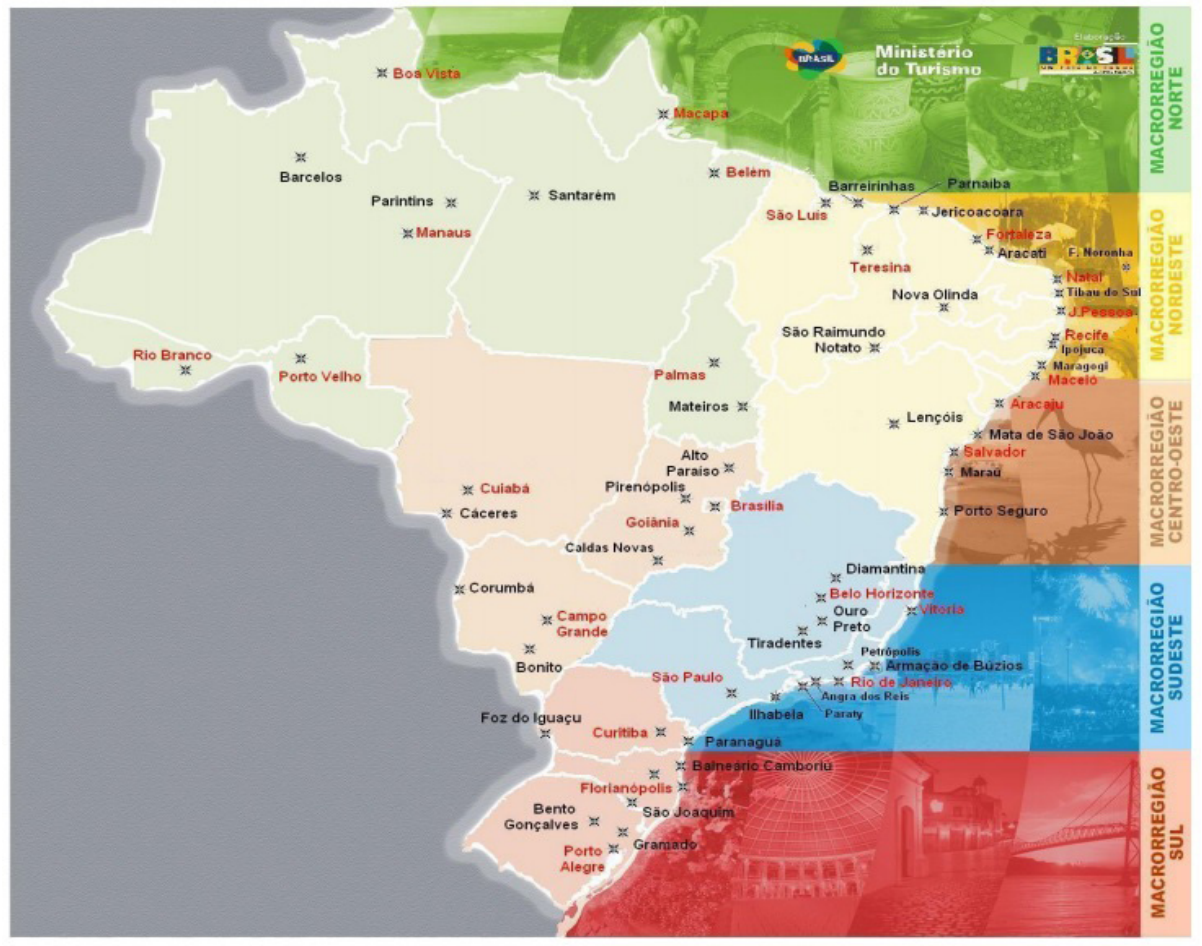

Fuente: Ministério do Turismo (2008). 
Análisis de la extinción de la política pública de destinos inductores del desarrollo del turismo regional en Brasil. Gestión Turística N 32 julio-diciembre 2019, pp 08-28.
Magnus Emmendoerfer

Fábia Tretin

Thiago D. Pimentel

Ma. Belén Zambrano-Pontón

Vale mencionar que, Minas Gerais es una unidad federativa de Brasil, situada en la región Sureste del país, sus dimensiones podrían ser comparables a Suecia, Francia, Japón y su economía equiparable a Israel, Irlanda, Chile o la Republica Checa (Emmendoerfer \& Soares, 2014).

El universo de investigación fueron los actores públicos y privados de turismo de los cuatro DIDTR del estado de Minas Gerais y del gobierno federal, específicamente del Ministerio del Turismo, conforme presentado en Tabla 2.

Tabla 2. Entidades y sujetos de la colecta de datos.

\begin{tabular}{|c|c|c|c|}
\hline Nivel & $\begin{array}{c}\text { Enti- } \\
\text { dad }\end{array}$ & Sujeto & Códigos \\
\hline \multirow{6}{*}{ 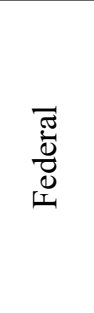 } & \multirow{6}{*}{ 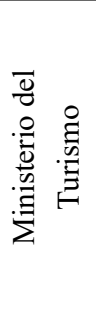 } & Burócrata Líder & E13 \\
\hline & & Burócrata Líder & E14 \\
\hline & & Burócrata Técnico & E15 \\
\hline & & Burócrata Técnico & E16 \\
\hline & & Burócrata Técnico & E17 \\
\hline & & Burócrata Líder & E18 \\
\hline \multirow{12}{*}{ 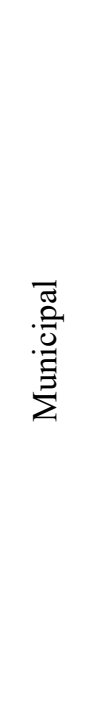 } & \multirow{2}{*}{ 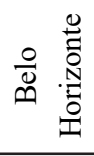 } & Gestor Burócrata Técnico - Belotur & E1 \\
\hline & & Técnico Privado - Belo Horizonte Convention \& Visitors Bureau. & E11 \\
\hline & \multirow{2}{*}{ 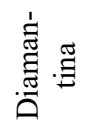 } & Gestor Privado - Circuito de los Diamantes & E2 \\
\hline & & Burócrata Técnico - Secretaria Municipal de Turismo & E3 \\
\hline & \multirow{4}{*}{$\begin{array}{l}\stackrel{0}{0} \\
0 \\
0 \\
0 \\
0\end{array}$} & Burócrata Técnico - Secretaria Municipal de Turismo & E4 \\
\hline & & Gestor Privado - Ouro Preto y Circuito del Oro C\&VB & E5 \\
\hline & & Burócrata Técnico - Asociación del Circuito del Oro & E10 \\
\hline & & Político Parlamentar & E12 \\
\hline & \multirow{4}{*}{ 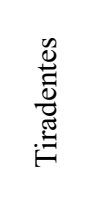 } & Gestor Privado - Asociación Empresarial de Tiradentes & E6 \\
\hline & & Gestor Privado - Circuito Turístico Trillas de los Inconfidentes & E7 \\
\hline & & Gestor Privado - Instituto Histórico y Geográfico de Tiradentes & E8 \\
\hline & & Gestor Burocrata Líder - Secretaria de Turismo y Cultura & E9 \\
\hline
\end{tabular}

Fuente: Elaboración propia. 
Para fines de esta investigación se optó por nombrar a los sujetos de investigación como burócratas líderes y burócratas técnicos, para mantener el anonimato de los entrevistados que fue garantizado en el Término de Consentimiento Libre y Esclarecido -TCLE, del sector público, nacional o municipal; así, también se utilizaron los términos Gestor y Técnicos para los pertenecientes a la iniciativa privada que participaron de la investigación.

Los datos colectados fueron tratados con la técnica de análisis de contenido temático. El tema fue definido como Extinción de un Proyecto Gubernamental. A partir de que los datos empíricos fueron cotejados y comparados con la teoría empleada, indicada en las referencias de este artículo, se observó datos peculiares, en el caso estudiado, que no estaban contemplados o que no estaban siendo tratados por la comunidad científica del área de gestión de las políticas públicas en el contexto del turismo. La presentación y la discusión de los resultados revelan con más detalles esas particularidades al respecto de la extinción de las políticas públicas. Se resalta que el rigor de la descripción y del análisis emprendido buscó triangular los datos empíricos con los datos secundarios de documentos y de naturaleza bibliográfica (teorización utilizada en este artículo) a fin de cuidar el rigor y calidad científica de esta investigación.

\section{Resultados}

En un primer momento y, a través de los documentos oficiales encontrados por datos documentales, fue percibido como un posible motivo para la extinción del proyecto en estudio, la creación de la categorización - política nacional de turismo vigente en el gobierno federal (Miniterio de Turismo de Brasil, 2019), en los documentos de la categorización, se intenta justificar la adopción de una nueva política, más amplia y más justa, dejando de priorizar solo 65 destinos y, como dice, que "[...] evolucionó y amplió su vista para todos los municipios contemplados en el mapa del turismo brasileño" (Ministerio de Turismo de Brasil, 2016, p.7, traducción nuestra), encajando como apunta Souza y Secchi (2015) dentro de las razones relativas a la solución y dentro de ella a que la política pública fue sustituida.

Pero, después de la visita y colecta de datos en el Ministerio del Turismo, fue identificado que la Extinción del Proyecto de los 65 DIDTR puede haber sucedido en dos momentos: una extinción para fines internos y otra para fines externos, la primera sucedió en el año 2011 y la segunda, cuatro años después, en el 2015. 
Para los dos casos, la extinción no fue comunicada debidamente a los municipios participantes del Proyecto 65 DIDTR, autores como Santos (2012) consideran la comunicación como una herramienta indispensable para la administración pública, que los entes del sector público tienen que aprovechar para divulgar o comunicar las acciones que están siendo realizadas por las instituciones, siendo también, una responsabilidad que, como funcionarios públicos, adquieren para informar a los involucrados en las políticas públicas y a la población en general sobre las inversiones de los recursos que están siendo pagados por la propia población y transparentar así, la gestión de la administración pública.

\section{Extinción para fines Internos}

Para fines de esta investigación, la extinción para fines internos corresponde a una decisión tomada por los actores involucrados en el proceso decisorio de esa política (MTur) y, que comunicaron esa extinción solamente en el nivel interno de la organización, no siendo exteriorizado para dominio público. Considerando el hecho de que no fue esclarecido para los destinos que el Proyecto había sido cerrado en 2011, aparentemente, para ellos solo ocurrió una interrupción en el año 2012.

Esa Extinción para fines Internos (dentro del MTur) sucedió en 2011, por cuenta de la "Operación Voucher" de la Policía Federal. Esta operación tenía el objetivo de combatir el desvío de recursos por medio de enmiendas parlamentarias. Esa operación fue comandada por la Policía Federal Brasileña, que resultó en la detención del Secretario Ejecutivo de Turismo (número dos del Ministerio), el Secretario Nacional de Desarrollo de Programas de Turismo y otros funcionarios del Ministerio (Globo G1, 2011).

Con esta operación policial casi todos los programas dentro del MTur fueron suspendidos, entre ellos, inclusive el Programa de Regionalización del Turismo $\mathrm{y}$, en el caso de los DIDTR, no fueron renovados los contratos para la ejecución en campo, tanto del estudio de competitividad, así como la monitoria a través del Sistema de Gestión del 65 destinos (SG65), que seguía todas las acciones de los destinos, recordando que fue realizado el estudio de competitividad en ese año 2011, pero que para el año 2012 no fue firmado nuevamente para su ejecución. Y fue en el año 2012, con el inicio de una nueva gestión dentro del MTur, que se tenía el interés de continuar con los análisis de los estudios de competitividad y fue iniciada una nueva negociación con la Fundación Getulio Vargas - FGV, para 
dar continuidad. La FGV fue la Institución del MTur para realizar las investigaciones en campo de los 65 destinos inductores y la emisión de los informes de los estudios de competitividad en esos destinos.

En 2013, las investigaciones continuaron en esos mismos 65 destinos, hasta el año 2015, quedando solamente esa acción como análisis, ya no como proyecto, ya que de acuerdo con los relatos de los entrevistados el proyecto de los destinos inductores acabó en 2011, pero para la sociedad y para los destinos, por lo menos en el caso de Minas Gerais, ese cierre no había sido comunicado, entonces, la extinción ocurrió para fines internos, apenas para conocimiento en el MTur.

De ahí la importancia de comunicar sobre los motivos de la extinción de un programa o proyecto de administración pública, según con el MTur, los motivos fueron varios y en la visión del Entrevistado E18:

(...) En 2010 ocurre el cambio de gobierno, aunque del mismo partido político, el enfoque de la política pública cambió [...], entonces el enfoque que se dá a la política pública de 2007 a 2010 fue uno, de 2011 a 2014 fue otro, eso está en los documentos de la política nacional de turismo, de 2007 a 2010 todavía se tenía los discursos de la inclusión social [...] de 2011 a 2014 se está hablando más en mercado, grandes eventos se está pensado en la Copa do Mundo, Olimpíada y, pensando otras cosas y, ahí no es solo esa transición de gobierno federal como un todo, fue, una transición tumultuosa del Ministerio del Turismo, hubo todo aquel problema relacionado con la transición del ministro, quedó un año prácticamente sin hacer ninguna gestión (2011) y, al final del año fue sustituido por otro, con denuncias de corrupción, entonces 2011 fue un año prácticamente perdido, 2011 fue un año que quedó parado, en 2012 comienza un esfuerzo del Ministerio del Turismo, mejorar la Gestión y eso inclusive interrumpió la mayoría de los convenios que eran el foco de las denuncias de corrupción, entonces paró proyectos de marca Brasil [...] y, ahí el segundo es la falta de resultados, en 2012, cuando se miraba para atrás se veía muy poco, un resultado concreto para evaluar, bueno no tenía nada concreto, los costos eran muy altos, se decidió dar continuidad con otro abordaje, el Sistema SG65 también era un sistema muy caro, los convenios también tenían un costo alto, además de las denuncias de corrupción, entonces todo el proyecto tenía un costo demasiado alto enfocado en una sola secretaria, y que era 
muy poco aprovechado por el Ministerio del Turismo (E18) (resaltado y traducción nuestra).

En ese sentido, en la visión del entrevistado E14 "los pilares" que sustentaban el proyecto acabaron, ellos ya no eran más posibles de ser contratados de la forma como eran contratados por el Ministerio del Turismo, entonces, la verdad, usted no tenía más pilares desde 2011. Los pilares fueron las acciones del proyecto DIDTR: 1. Estudio de Competitividad a cargo de la Fundación Getulio Vargas; 2. Gestión de los Destinos a través de la capacitación a los grupos gestores en los 65 destinos inductores, responsabilidad del Instituto Marca Brasil y, 3. La monitoria a través del sistema de Gestión SG65, a cargo de la empresa Modulo de Tecnología.

Siendo así, fueron varios los motivos para el fin del proyecto 65 DIDTR, con todo no se explica el porqué de no haber sido comunicado el final del proyecto y la continuidad de apenas una determinada acción (Estudio de Competitividad) en cooperación técnica con la FGV.

\section{Extinción para fines Externos}

De esa forma, el cierre para fines externos, se aproxima de la extinción de políticas públicas como había sido tratado hasta hoy, aproximándose así de la definición propuesta por Secchi (2013), que contempla el fin de todas las acciones de la política, en este caso del proyecto que sucedió en 2015, cuando fue elaborado el último Estudio de Competitividad en los destinos y los municipios sintieron la falta de esas investigaciones por los informes que recibían al final del año, pero, que también, no fue comunicado debidamente esa extinción, existiendo una falta de transparencia por parte del MTur como órgano encargado de la Gestión dentro de la Administración de la Política Nacional de Turismo en Brasil.

Siendo así, esa extinción, también sucedió por varios motivos, partiendo del cambio de administración, siguiendo con la reestructuración del MTur en 2015, reasignando la competencia por el Índice de Competitividad al Departamento de Estudios e Investigaciones, un departamento que en aquella época tenía un equipo restricto y un presupuesto decreciente y limitado y, que también no tenía el interés de continuar con los estudios. Siendo así, el principal motivo la falta de recursos y de interés en dar continuidad, que según el entrevistado E18: 
(...) la verdad, hubo un cambio de administración en el Ministerio del Turismo; así, se cambió la estructura del Ministerio en 2015 y, se redistribuyó las competencias en el Ministerio del Turismo. El programa de los 65 destinos había acabado y, lo único que sobró eran los estudios de Competitividad, el índice no era una acción propia para los destinos, es un índice y ahí fue realizado en el año 2015, en esa redistribución de competencias, fue reasignar esa atribución para el área de estudios e investigaciones, que en teoría tenía sentido, el índice no es una política de restructuración de destinos, y es solo un índice, solo una investigación, entonces esa área de estudios e investigaciones debería cuidar de esa investigación y de ahí esa área debía subsidiar esas informaciones, tuvo sentido desde el punto de vista teórico, pero en la práctica tenía un equipo muy limitado en la época, que no atendía el conjunto de investigaciones que tenían que contratar, pero más grave todavía, ellos no tenían recursos, entonces ellos tenían un presupuesto de doce millones para la investigación, ese presupuesto fue reduciendo, en el año 2015 ese presupuesto ya era de 7 millones y era una investigación más para hacer que costaba un millón y medio y no dieron para ellos, ni ese millón y medio, entonces de 12 millones cayeron para 7 , entonces, en la práctica se mató el estudio, operacionalmente, ya no era posible, por otro lado también, existía también el punto de vista técnico, dentro del departamento de estudios e investigaciones no tenían apoyo suficiente, los técnicos, el coordinador general, no tenían interés en ese índice, y no tenía ni de la administración del MTur el apoyo suficiente (E18) (resaltado y traducción nuestra).

De ese modo, se evidencia que se perdió el interés en los resultados que el índice de competitividad podría presentar, pues ellos no eran más considerados como prioridad para el Ministerio del Turismo, así como, la cuestión económica ser un factor limitante para que las investigaciones continúen siendo realizadas por la FGV. El entrevistado E15 cree que no hubo comunicación, así coloca lo siguiente:

(...) no fue comunicado de forma oficial, porque hasta hoy, todavía nos preguntan qué sucedió con los 65 destinos, pero cuando comenzó la categorización tuvimos cuidado de hacer un capítulo explicando que no se está trabajando más priorizando apenas 65 destinos, el Ministerio tiene un mapa de turismo con más de 3000 municipios y, que en la medida de lo posible va intentar atenderlos de la forma que más necesitan, entonces 
dejó de atender apenas los 65 para intentar hacer un recorte mayor [...] con la categorización priorizaríamos realmente ese ciclo (E15) (resaltado y traducción nuestra).

Contrariamente con otras declaraciones, el Entrevistado E14, considera que "fue comunicado, eso ya se venía comunicado siempre, pero oficialmente fue identificado, con la publicación de la categorización (Ministério do Turismo, 2016), cuando se comunicó la categorización se colocó la información de las razones de ser retirado, de no ser priorizado más". En la visión del entrevistado E16, los motivos fueron que:

(...) la idea original fue menguando hasta quedar solo el estudio de competitividad y fueron cuestionados por instancias superiores, para qué están realizando esos estudios, qué información saco de ese estudio, es una información de 65 municipios, el Brasil tiene 5570 municipios, y considera que esos municipios no son representativos del turismo nacional, si se ve con mucha buena voluntad las capitales son más, en un principio cómo distinguir el flujo de turistas de ocio por los de negocios, qué está midiendo, y peor para qué está utilizando (E16) (traducción nuestra).

Delante de eso, se evidencia que ni dentro de propio MTur quedaron claros los motivos para el cierre para fines internos en el proyecto en el año 2011 y después, en el año 2015, para fines externos.

\section{Percepciones de la extinción del proyecto de los destinos inductores na- cionales}

Fue preguntado para los gestores de la administración pública municipal, de los cuatro destinos inductores nacionales de Minas Gerais, qué consideraban como posibles motivos para el cierre de la política pública DIDTR, se preguntó, en ese sentido, pues se percibió que ellos no fueron comunicados, inclusive, muchos de ellos no conocían el hecho de que el proyecto había sido extinto y hasta lamentaban el final de las investigaciones de los índices de Competitividad, por considerar que esas investigaciones dieron soporte y auxilio por medio de informaciones importantes, pues consideraban que el proyecto contribuyó para conocer la situación de su municipio. 
Como muestra la Tabla 3, los autores presentaron las principales razones para la extinción de las políticas públicas; En ese caso específico de los DIDTR fue posible identificar otras razones de extinción de las políticas públicas, que fueron colocadas en negritas para diferenciarlas de las mencionadas por Souza y Secchi (2015).

En ese sentido, se evidenció que la mayoría de los entrevistados tanto del nivel federal, como en el nivel municipal y los investigadores, consideran como los principales motivos para la extinción de la política pública en estudio, las razones relativas al ambiente político, siendo la limitación de recursos financieros dentro del MTur el motivo con mayor percepción, seguido por el cambio en la Administración, en el Gobierno y la ideología política.

Sumado a eso, fue posible identificar en esta investigación otras percepciones adicionales a las encontradas en la literatura sobre extinción y que fueron encajadas dentro del ambiente político (en negritas), ellas fueron: ineficiencia organizacional con la mayor parte de las percepciones, corrupción, en segundo lugar, de los nuevos motivos o percepciones. Luego fue percibida la falta de planificación, así como la falta de interés en dar continuidad al proyecto, según la percepción de los entrevistados, que llevaron al MTur a extinguir el proyecto.

Así también fue percibido que las razones relativas a la solución, y entre ellas las percepciones de la Política Pública sustituida y la Política Pública percibida como ineficaz, en esta razón también fue posible identificar (en negritas) la falta de resultados del proyecto con tres percepciones cada una de ellas.

Adicional a eso, el motivo de las razones relativas al problema que tuvo una mayor percepción fue el cambio en el entendimiento de la naturaleza del problema. Se adicionó otra percepción (en negritas), adicional de las propuestas por Souza y Secchi (2015) que tiene relación con la resolución del problema, esas percepciones fueron tanto del gobierno federal, municipal y de los investigadores.

En esta investigación se destaca, la falta de comunicación evidenciada entre la institución de la administración pública encargada de la formulación de las políticas públicas de turismo en Brasil y los municipios involucrados en esas políticas. 
Análisis de la extinción de la política pública de destinos

Tabla 3. Razones y percepciones para la extinción de la política de los DIDTR

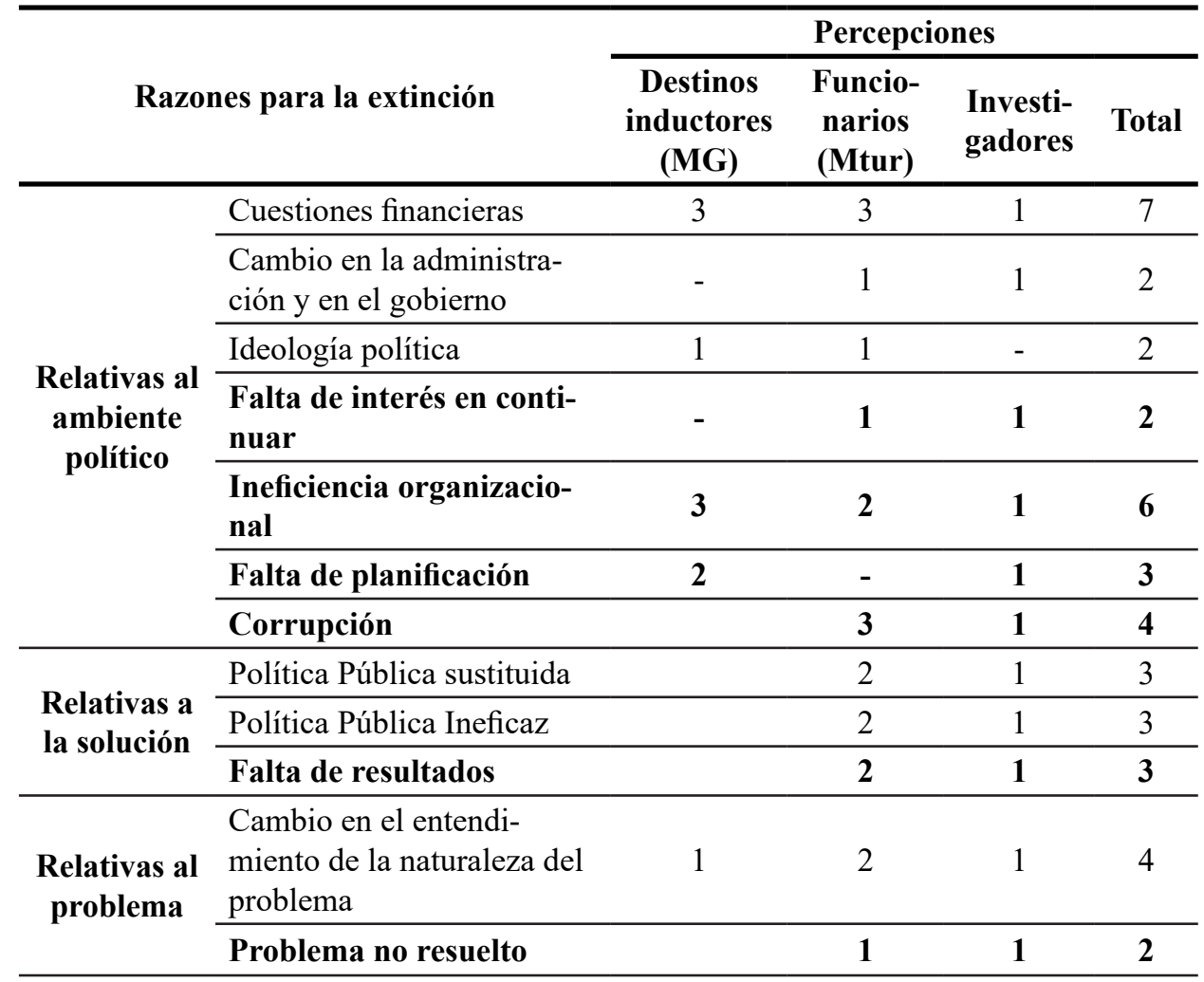

Fuente: Datos de la investigación. Adaptado y elaborado a partir de Souza y Secchi (2015).

Y en ese sentido, la atribución por la comunicación de las políticas públicas de turismo entre el Gobierno Federal y Municipal, le correspondía al MTur, como órgano coordinador y encargado de la Gestión del turismo en Brasil, el cual debía informar a los municipios, la extinción del proyecto de los destinos inductores, y contrariamente, fue percibido que existió una desinformación y una falta de transparencia, pues en los municipios no se conocían las razones que pudieron haber influenciado y acabado en el cierre del proyecto en estudio.

Siendo precisamente, la principal fragilidad de la extinción la falta de comunicación y transparencia, pues no fue comunicado a los destinos involucrados ni a la 
sociedad que el proyecto acabó en el año 2011, ya que aparentemente, continuó hasta el año 2015 con la acción más emblemática y visible que tenían los destinos inductores, los Estudios de Competitividad.

Como un aspecto positivo, resultado del proyecto DIDTR, se destaca la serie histórica de informaciones relevantes provenientes de los estudios de competitividad para cada uno de esos 65 destinos inductores, independientemente de la continuidad o no del proyecto, pues esa serie histórica recabó informaciones importantes, que en los destinos inductores (por lo menos en el caso del estado de Minas Gerais) continua contribuyendo, para la formulación e implementación de políticas públicas en los municipios del estado de Minas Gerais, siendo un aspecto relevante de mencionar.

Así, una fragilidad de las políticas públicas, podría ser el cambio de Gestión dentro de la Administración Pública en todo nivel, sea federal, estadual y municipal, por el hecho de que, a veces, son descartadas políticas públicas, sin haber sido realizadas, por lo menos, un análisis de resultados que permita comprender qué sucedió y sin comunicar las decisiones ya tomadas o que van a ser tomadas, por lo menos a los involucrados en esas políticas.

Esta investigación permitió evidenciar que no existen estudios en Brasil que analicen la extinción del proyecto de los 65 Destinos Inductores para el Desarrollo del Turismo Regional, siendo varias las razones que motivaron al Ministerio del Turismo en Brasil a extinguir el proyecto de los Destinos Inductores, en el año 2011, y, posteriormente, el cierre de las investigaciones del Índice de Competitividad, en el año 2015, siendo ellas, principalmente, la falta de recursos presupuestarios, ineficiencia organizacional por falta de cumplimiento de los objetivos propuestos, problemas de corrupción que involucraban al MTur en la época de la implementación del proyecto, el cambio del entendimiento del problema y de Gestión, evolucionando, así, con una nova política que ahora, abarca más municipios y que necesitan de una inversión menor de recursos, aunque, enfocados, principalmente, en el desempeño de la economía de esos destinos turísticos, como aparenta ser la premisa de las nuevas políticas públicas nacionales de turismo en Brasil. 


\section{Conclusiones}

Esta investigación permitió identificar que no existe una única forma de extinción, pues en la política analizada, fue identificado un cierre más limitado a fines internos, dentro de la organización (2011), llegando, solo cuatro años después, a la extinción para fines externos (2015), teniendo, así, más de un tipo de extinción. Se infiere que la dimensión temporal auxilió en la identificación y en la búsqueda del entendimiento del caso estudiado, bien como permitió argumentar que el proceso de extinción puede no ocurrir en un único momento en términos de gestión en organizaciones públicas en un plano intergubernamental.

En cuanto a las formas de extinción se puede decir que el Proyecto 65 DIDTR fue extinto repentinamente en consecuencia de la "Operación Voucher" envolviendo corrupción, limitando el uso de recursos y requiriendo una gestión de crisis dentro del MTur (Silva \& Costa, 2018), así como el cambio de dirección dentro de la Administración Pública teniendo como resultado una ineficiencia dentro de la organización, principalmente.

Se destaca la importancia de la comunicación y de la transparencia que, en este caso específico de los DIDTR, no existió entre el Gobierno Federal y Municipal y más específicamente, por parte del MTur, como institución que dirige la gestión del turismo en Brasil, transparentando e informando a los municipios el fin de la política de los destinos inductores.

Las razones contempladas en la teoría sobre el cierre fueron tres: Relativas al ambiente político y las percepciones encontradas fueron: Cuestiones Financieras, Cambio en la Administración y en el Gobierno e Ideología política; la segunda razón fue la Relativa a la solución y dentro de ella los motivos encontrados fueron: Política pública sustituida y política pública ineficaz y la tercera razón relativa al problema, estando en ella: el cambio en el entendimiento de la naturaleza del problema.

Así, las nuevas percepciones o motivos encontrados fueron: la Falta de interés de continuar con la intervención gubernamental, Ineficiencia Organizacional, Falta de Planeamiento y Corrupción; ellas dentro de las razones relativas al ambiente político; Dentro de las Relativas a la Solución fue encontrado: la Falta de Resultados y, por fin las Relativas al problema: el Problema no resuelto. 
La contribución de esta investigación está dada en la replicabilidad, ya que puede ser aplicada en nuevos estudios y nuevos contextos sobre Políticas Públicas, Gestión, Administración Pública y Gobernanza Pública, tanto en Brasil como en el exterior, considerando la importancia que ellas tienen, sea en la forma de programas o proyectos para la población y, así comprender cuáles son los motivos del cierre de esas políticas, entendiendo lo que ellas representan en términos de nuevas inversiones, considerando que esas intervenciones gubernamentales son fruto de recursos provenientes de contribuciones que son pagadas por la propia población, que se debe exigir aún más responsabilización cuando se tienen muchos problemas de desigualdades sociales como en la América Latina.

Finalmente, esta investigación revela nuevas oportunidades de investigaciones teóricas y empíricas en relación a la fase de extinción de proyectos, programas, políticas gubernamentales y públicas que todavía ha sido sombreada, olvidada o subvalorizada en los estudios en el área de gestión pública del turismo tanto en Brasil como en el exterior.

\section{Agradecimimentos}

Agradecemos el financiamiento de Conselho Nacional de Desenvolvimento Científico e Tecnológico - CNPq (processos 429443/2016-1; 309363/2019-5), Fundação de Amparo à Pesquisa do Estado de Minas Gerais - FAPEMIG (processo APQ-01870-15; PPM-00049-18) y Coordenação de Aperfeiçoamento de Pessoal de Nível Superior - CAPES (Processo 001), Brasil.

\section{Referencias}

Araújo, L., \& Rodrigues, M. D. L. (2017). Modelos de análise das políticas públicas. Sociologia, problemas e práticas, 83, 11-35. http://dx.doi. org/10.7458/SPP2017839969.

Ministério de Turismo de Brasil (2008). 65 destinos indutores do desenvolvimento do turismo regional. Recuperado de http://www.turismo.gov.br/sites/ default/turismo/convenios contratos/downloads_convenios/65 DESTINOS RELACAO.pdf. [Versión original en portugués]. 
Análisis de la extinción de la política pública de destinos inductores del desarrollo del turismo regional en Brasil.

Gestión Turística N 32 julio-diciembre 2019, pp 08-28.
Magnus Emmendoerfer

Fábia Tretin

Thiago D. Pimentel

Ma. Belén Zambrano-Pontón

Ministério de Turismo de Brasil.(2009). Estudo de competitividade dos 65 destinos indutores do desenvolvimento turístico regional - relatório Brasil. Brasília, DF: Ministério de Turismo. Recuperado en http://www.turismo.gov.br/sites/default/turismo/o ministerio/publicacoes/ downloads publicacoes/MIOLO 65xdestinosx_revisao4set.pdf

Ministerio de Turismo de Brasil (2016). Categorização dos Municípios das Regiões Turísticas do Mapa do Turismo Brasileiro - Perguntas e Respostas. Brasília: Ministério do Turismo. Recuperado en http://www. turismo.gov.br/sites/default/turismo/o ministerio/publicacoes/downloads_publicacoes/Perguntas_respostas_Categorizacao_2016.pdf

Ministerio de Turismo de Brasil(2019). Categorização dos Municípios das Regiões Turísticas do Mapa do Turismo Brasileiro - Perguntas e Respostas. Brasília: Ministério do Turismo. (Trabajo original publicado en Agosto de 2019). Recuperado en http://www.regionalizacao.turismo. gov.br/images/conteudo/Perguntas_espostas_Categorizacao_2019.pdf

Castilho, C. J. M., \& Selva, V. S. F (2012). Turismo, políticas públicas e gestão dos ambientes construidos. Recife: UFPE.

DeLeon, P. (1977). Public policy termination: an end and a beginning, Policy Analisys, 6(1), 1-38.

Emmendoerfer, M. L. y Soares, É. B. S. (2014). Análise do desenvolvimento turístico e da Denominação Designada pelo Governo para qualificar cidades como destinos indutores do turismo em Minas Gerais, Brasil. Revista Turismo \& Desenvolvimento, v.3, n 21/22, p.139-151.

Frey, K. (2009). Políticas públicas: um debate conceitual e reflexões referentes à prática da análise de políticas públicas no Brasil. Planejamento e politicas públicas, 21, 211-259.

Giuliani, M. (2005). Policy termination. In G. Capano, G., \& M. Giuliani (orgs.) Dizionario di Politiche Pubbliche (pp.290-291). Roma: Carocci. 
Análisis de la extinción de la política pública de destinos inductores del desarrollo del turismo regional en Brasil.

Gestión Turística Nº 32 julio-diciembre 2019, pp 08-28.
Magnus Emmendoerfer

Fábia Tretin

Thiago D. Pimentel

Ma. Belén Zambrano-Pontón

Globo G1 (2011, agosto 11). Entenda a Operação Voucher, da PF, que prendeu o número 2 do Turismo. Brasília: Globo G1. Recuperado en http://glo. $\underline{\mathrm{bo} / \mathrm{nBiiVz}}$

Lasswell, H. D. (1956). The decision process: Seven categories of functional analysis. College Park, MA: University of Maryland.

Lynn, L. E., \& Gould, S. G. (1980). Designing public policy: a casebook on the role of policy analysis. Santa Monica, CA: Goodyear Pub.

Santos, E. G., Jr. (2012). A importância da comunicação na gestão pública e sua influência e relações com os interesses públicos, políticos e transparência governamental: o caso da prefeitura de Castro-Paraná. Publication UEPG: Ciencias Sociales Aplicadas, 20(2), 193-202. http://doi. org/10.5212/PublicatioCi.Soc.v.20i2.0006

Secchi, L. (2013). Políticas Públicas: conceitos, esquemas de análise, casos práticos. São Paulo: Cengage Learning.

Silva, B. R., \& Costa, H. A. (2018). Gerenciamento de Crises no Setor Público e suas Influências sobre a Administração: O Caso da Operação Voucher no Ministério do Turismo. Revista do Serviço Público, 69(2), 89-115. https://doi.org/10.21874/rsp.v69i2.1825

Souza, Y. H., \& Secchi, L. (2015). Extinção de políticas públicas. Síntese teórica sobre a fase esquecida do policy cycle. Cadernos Gestão Pública e Cidadania, 20(66), 75-93. http://dx.doi.org/10.12660/cgpc.v20n66.39619

Subirats, J. (1989). Análisis de politicas públicas y eficacia de la administración. Madrid: Instituto Nacional de Administración Pública. Recuperado en https://pdfs.semanticscholar.org/ e 7f6/fed7450301cce143b62182fe120d491fab80.pdf 